\title{
Advocacy, Collaboration, and Intervention: A Model of Distance Special Education Support Services Amid COVID-19
}

\author{
Janice K. Frederick ${ }^{1}$ - Ginger R. Raabe ${ }^{1}$. Valerie R. Rogers ${ }^{1}$ (D) Jessica Pizzica ${ }^{2}$ \\ Published online: 14 August 2020 \\ (C) Association for Behavior Analysis International 2020
}

\begin{abstract}
The COVID-19 pandemic has significantly impacted the everyday lives of many individuals across the globe. The school closures across the majority of the United States have presented administrators, educators, and behavior analysts with the unprecedented task of deciding how best to teach and support our students, especially those accessing special education services. The current article describes the steps our program took, in light of school closures, to advocate for and ultimately create and implement a model that allows special education students to access the behavior-analytic educational supports they had received on campus (e.g., Board Certified Behavior Analyst and paraprofessional support) in a novel and remote manner. We share details regarding the advocacy and collaboration process, as well as the distance special education support model itself, in hopes that similar processes and models can be implemented across geographical locations to assist special education students in accessing their educational and behavioral supports in a meaningful way throughout current and future school closures.
\end{abstract}

Keywords Applied behavior analysis · California Department of Education · COVID-19 · Nonpublic agency · Special education · Telehealth

In response to the 2019 coronavirus disease (COVID-19) outbreak, schools across the world have been closed for multiple weeks as an emergency measure to prevent the spreading of the infection. At the time of publication, in the United States, school closures due to COVID-19 have impacted at least 124,000 public and private schools, affecting at least 55.1 million students (Peele and Riser-Kositsky, 2020). The current

\section{Editor's Note}

This manuscript is being published as part of a series of emergency publications designed to help practitioners of applied behavior analysis take immediate action to adjust to and mitigate the COVID-19 crisis. This article was submitted on April 10, 2020, and received final acceptance on May 29, 2020. The views and strategies suggested by the articles in this series do not represent the positions of the Association for Behavior Analysis International or Springer Nature.

Electronic supplementary material The online version of this article (https://doi.org/10.1007/s40617-020-00476-1) contains supplementary material, which is available to authorized users.

Janice K. Frederick

janice.frederick@abrite.org

1 The ABRITE Organization, 749 37th Ave., Santa Cruz, CA 95062, USA

2 Santa Cruz City School District, Santa Cruz, CA, USA pandemic has resulted in an immediate cessation in education as we have known it and a subsequent push to "distance learning" via guidance from federal, state, and local educational agencies. Distance learning has been defined as instruction in which the student and instructor are in different locations. This instruction may take a variety of different forms including, but not limited to, interacting through the use of computer and communications technology, live instruction involving audio and/or video engagement between a student and instructor in an individualized or group format, printed materials provided to the student, and written feedback provided to the student related to completed assignments. Although it is not always required, many distance learning plans that school districts have adopted appear to rely heavily on instruction provided via online engagement and video-based instruction (California Department of Education, 2020).

Local education agencies and school districts are facing unprecedented challenges as they develop methods, train their teams, and introduce distance learning to their students. School districts are further strained as they plan for serving students with disabilities. These students are especially vulnerable to regression when service is removed, reduced, or modified and often receive a number of services as part of their individualized educational programs (IEPs). According 
to the National Center for Education Statistics (2020), nearly 6.7 million students in the United States receive special education services under the Individuals With Disabilities Education Act (IDEA, 2004). A critical component of IDEA is to provide students with disabilities a free and appropriate public education (FAPE), and the U.S. Department of Education has clearly indicated that school districts are still required to provide FAPE during this pandemic (U.S. Department of Education, 2020), including the implementation of IEPs to the maximum extent possible. School districts have been encouraged to utilize distance learning models to serve students with disabilities and to support these models with other forms of service delivery if needed. Students with IEPs may require various support services, including specialized academic instruction, special individualized support, behavior intervention development and supervision, counseling, and/or additional therapies such as speech, occupational, physical, and vision. Again, per federal and state mandates, students should continue to receive these services to the fullest extent possible during school closures. Students' goals and service levels must be considered when identifying methods and rates of service delivery during this unprecedented period. Once methods and rates are established and introduced, service providers should demonstrate full commitment to initial and ongoing data-based assessment and analysis so these can be modified as needed.

Although school districts seem to be in agreement that the majority of services outlined on the student's IEP must be implemented to some degree, there seems to be a less uniform position related to individualized behavior support services or the one-to-one support often provided by a registered behavior technician, behavior interventionist (BI), or instructional assistant trained in applied behavior analysis (ABA). These essential paraprofessionals may be employed internally by the school district or by a nonpublic agency (NPA) working collaboratively with the school district to support students. In addition to the individualized behavior support services provided by paraprofessionals, students often receive supervision services by a Board Certified Behavior Analyst (BCBA). With respect to these services, particularly when the services are provided via an NPA, there are inconsistencies across school districts regarding the continuation of service and the level at which it is provided or even feasible during the periods of closure. Given the role that these service providers play in enabling students to access their educational program, it seems irrefutable that these services should continue to the maximum extent allowable during this arduous circumstance. These services are critical at a time when students and their parents are placed under instructional conditions that are novel, fraught with accessibility issues, and estimated to extend for the next several months. Parents have been put in the position to serve as the primary implementor of their child's education, and this brings unique challenges for students with special needs and their parents. BCBAs are in a position not only to support students' access to academic and behavioral components of individualized instructional programs, but also to support the team in measurement and analysis of student engagement and progress under these new learning conditions. It is simply unjust to make assumptions that the student's caregiver would serve as the sole facilitator of the student's educational program under these conditions. BCBAs have the skills and an extensive research literature to support methods and strategies that can be shared with and taught to parents to make distance learning easier.

During the current pandemic, ABA practitioners have attempted to implement telehealth practices across varying service models (e.g., supervision, parent training, direct oneto-one treatment), despite the fact that this format is new to most ABA providers. An expanding body of literature on telehealth models of ABA has emerged in recent years, including research on parent training, consultation, and case supervision provided via telehealth (Ferguson, Craig, \& Dounavi, 2019; Tomlinson, Gore, \& McGill, 2018). Only more recently have authors begun to provide guidance on the implementation of telehealth models involving remote intervention provided by a BI. In the first article in the Behavior Analysis in Practice emergency publication series related to the global COVID-19 pandemic, Rodriguez (2020) provides a tentative framework for assessing the appropriateness of different telehealth models in the context of insurance-funded, medically necessary treatment. These considerations may also be applied to the implementation of behavior-analytic "distant learning" models within an educational framework. Behavior analysts are in a prime position to assist special education administrators in the creation and implementation of distant learning given the progressively available information related to telehealth service provisions.

There is so much power and possibility when members within a given team, such as a school district and an NPA, can collaborate and work together to establish guidelines and parameters for supporting the students and families they serve. Positive outcomes and equity can be established for all students when agencies such as NPAs are involved in the development and discussion related to the continuation of services for their students. Effective collaboration within and across agencies supporting special education students is likely to result in the most meaningful educational benefit. At times of unexpected hardship, collaboration, within and especially across agencies, can be a difficult commitment. We wanted to share the experience of our organization, which provides supports to special education students via our NPA, in navigating and securing continued service for our students via a model for distance educational and behavioral support. We were able to initiate support for numerous students via a model developed under a very abbreviated timeline that resulted in a minimal break in service, a reported reduction in stress and an 
increased sense of support by students and parents, and an immediate increase in student academic task completion and concomitant reductions in behavioral excesses. It is our hope that sharing our model, which incorporated advocacy, successful collaboration, and clinically sound practices, can assist school districts, external agencies, and service providers in facilitating increased access for students with developmental disabilities.

\section{Framework for Distance Support: Individualized Behavior and BCBA Support Services}

Immediately upon news of a brief closure of all county schools, we initiated the creation of a distance model for student support based on the assumption that closures were likely to extend well beyond the dates of the initial shutdown. First, we understood that the model would necessitate distance support, given shelter-in-place guidelines in the county. Second, our BCBAs initiated immediate advocacy related to the continuation of service for each student and proposed that students initially receive at least $15 \mathrm{hr}$ weekly ( $3 \mathrm{hr}$ daily) of individualized support, as well as BCBA supervision at the same frequency of service outlined in their IEPs. During these early weeks, the lack of standardization and guidelines for distance learning seemed to be breeding confusion and inconsistencies; therefore, a clear framework that included daily support hours was suggested as a starting point to provide some guidance. Considerable advocacy and collaboration were required to secure these services for students. The school districts in contract with the NPA were in triage mode, and considerable discussion was required to establish agreement between the school districts and NPA related to the interpretation of what the implementation of students' IEPs to the "fullest extent possible" could look like under a distance model. More specifically, some school districts took the position that little to no behavioral support services could be provided at a distance. The collaboration between the school districts and NPA involved a review of historical data, a review of parent survey data related to support needs and concerns (collected immediately upon notice of school closure), a discussion of and recommitment to shared values as a collaborative team, and an extensive breakdown of proposed distance model components and a vision for what support sessions would involve. The model was comprised of five components:

1. a parent interview and accessibility assessment,

2. BCBA program preparation,

3. BI training in distance support strategies,

4. distance support intervention sessions, and

5. BCBA supervision and parental support (see Table 1).
Preliminary Implementation of the Distance Special Education Support Model

\section{Participants}

The distance support model was introduced the week immediately following school closure in the county. We are currently serving 24 students who were previously receiving support via an NPA in a public school placement. Each student served by the NPA's distance support model has a current IEP and an attached behavior intervention plan that addresses one or more behavioral excesses. Students' IEPs prescribed services including, but not limited to, behavior intervention implementation (BII) and BCBA-level supervision. Students' IEPs mandated BII support for the duration of their school day, as well as BCBA supervision ranging from 90 to $360 \mathrm{~min}$ per week. Students are eligible for special education services under a variety of different educational categories, including autism, intellectual disability, emotional disturbance, and other health impairments. Students range in grade from 1st to 10th and in age from 6 to 16 years. A majority of the students are placed in a general education setting and receive $80 \%$ or more of their services in that setting. The remaining students are placed in special day classrooms.

\section{Setting}

During the initial week of the distance support model, interventionists worked from one clinical location. The model was introduced at a single clinical site (i.e., as opposed to clinicians' private homes) to support rapid problem resolution by BCBAs particularly with respect to administrative and technology-related items on the end of the student's caregiver or the interventionist. To maintain safety at the clinical site, an extensive list of safety precautions was put in place, including solitary entry across multiple building entrances, social distancing, repeated and deep sanitization of the site during and following sessions, and storage of shoes and personal items prior to entry into the building. Interventionists worked from independent distance support stations located 10-20 ft from one another, and problem resolution by BCBAs was primarily conducted via videoconferencing and on-site observations and equipment checks. Following 5 days of site-based distance support and the passing of a procedural check related to access to secure workstations, interventionists were transitioned to providing the distance support sessions from their homes. Since this time, additional interventionists have been trained to support students in the distance support model, and this training has been conducted successfully using digital platforms and has not required working from a uniform location.

\section{Equipment}

Each interventionist's workstation was equipped with a device that included an electronic data-tracking application (e.g., 
Table 1 Overview of the Distance Education Support Model and Its Essential Components
The BCBA conducts an open-ended interview.

The BCBA completes a functional assessment interview with the parents.

The BCBA sends out a survey on technology at home for problem resolution.

Create a workstation video.

BCBA Program Preparation

The BCBA is assigned to examine the IEP for student services and goals.

Create modifications to any curriculum, data collection method, and reinforcement or prompting strategies.

Create sessions checklists that outline session structure (Appendix D).

The BCBA probes basic prerequisite skills for risk assessment (Appendix C).

Behavior Interventionist (BI) Training

Student-BI pairings are assigned.

Group-based training in rapport building and prompting and reinforcement strategies via technology are held.

Training materials are developed: distance learning structure supports, developing a workstation, positive statement protocol, prompting recs, technology tips, and telepractice quick tips.

Give weekly support and feedback.

Once the BI demonstrates mastery in a controlled condition and his or her workstation is approved, the BI can transition to the home workstation.

Weekly ongoing training via the virtual platform is held.

Distance Support Intervention Sessions

Sessions are held for $3 \mathrm{hr}$ per day, 5 days per week.

Sessions include rapport building and positive statement timing, structured teaching, and check-ins on academic work and targeting of replacement skills.

BIs are given access to Google Classrooms utilized by the teacher.

BIs are also supported during virtual sessions with other service providers (e.g., speech, occupational therapy, resource).

BCBA Supervision and Parent Support

The BCBA conducts observations to ensure treatment integrity.

BCBA modifies behavior protocols and tracks data, phase changes, and all reinforcement protocols.

The BCBA guides communications between the parents, BI, teacher, case manager, and special service providers.

Video content is created by the BCBA to support students and their parents.

Video content includes creating a workstation, creating a routine and schedules for instructional activities, prompting hierarchies, shaping requests for breaks and assistance, and antecedent strategies for undesirable behavior.

Parents are given access to videos and have weekly parent training with the BCBA.
Catalyst), as well as applications for virtual meeting platforms and Google Classroom. Students accessed distance support sessions using a Chromebook, many of which were provided by their district, and/or a handheld device such as an iPad or Kindle. Interventionists and students had secure Wi-Fi connections.

\section{Procedures}

During the initial week of school closure, students were not provided with educational services at the discretion of the school districts. According to the California Department of Education, if a district closes its schools, in this case, to slow or stop the spread of COVID-19, and does not provide any educational services to the general student population, it is not required to provide services to students with disabilities during that same period of time. During this first week, the NPA examined students' IEPs to determine the means by which IEP goals could be addressed via BII and BCBA services to the "fullest extent possible." This analysis was used to create the general framework underlying the distance support model. This week was also utilized to meet repeatedly with special education administrators to discuss the proposed model, advocate for the implementation of services, and plan for the initiation of service.

Parent interviews Following approval by school district administrators, BCBAs employed by both the school district and 
NPA contacted the parent(s) of each student assigned to their caseload and conducted an open-ended interview that included questions related to the student's response to academic work presented by the parent and the behavioral challenges the student presented during these times (for students already assigned). Parents were also asked about what educational and/or behavioral supports they felt would be beneficial during the school closure. Interview data were summarized and shared with the Special Education Local Planning Area Director and Special Education Directors in an attempt to secure support for the distance support model.

A follow-up meeting was conducted with most parents via phone or Zoom, during which the BCBA conducted an openended functional assessment interview (Hanley, Jin, Vanselow, \& Hanratty, 2014) related to the undesirable behaviors the parent reported to observe, particularly during academic work periods. Parents were provided with an electronic survey related to their ability to access technology, including access to equipment and the Internet; their ability and preferences related to virtual platforms for meetings and sessions; and the space available for the student to complete work and participate in distance learning sessions. Following the initiation of support sessions, parents and students were surveyed to learn more about how they were doing in the context of school closures and their specific needs and requests (see Appendices A and B). Data obtained through these methods were utilized to further build out the model for distance support.

BCBA program preparation The BCBA supervising the student's services examined the student's IEP goals with a focus on those goals for which the BCBA served as the responsible party. These goals tended to focus on behavior reduction and the acquisition of positive replacement skills, including, but not limited to, appropriate mands for assistance, breaks, and attention; following multiple-step instructions; turn taking; waiting; reciprocal communication; attending to a speaker; task initiation; social problem solving; goal setting; and planning. The majority of students were placed in general education classrooms, and, therefore, opportunities to work on positive replacement skills most often occurred in the context of naturally occurring opportunities throughout the school day. The conditions of the distance model afforded the opportunity to target these skills in a more contrived preparation, allowing for repeated presentations within a single session. BCBAs conducted a brief assessment of the student's prerequisite skills required for distance support (see Appendix C). BCBAs completed needed programming for how these skills would be addressed in an individualized distance support session, and targets and measurement parameters were programmed into the student's digital tracking application. The BCBA made modifications to reinforcement, prompting, and behavior intervention protocols to allow for implementation under the conditions of the distance support model and customized distance intervention sessions to meet the needs of the individual student.

BI training in distance support intervention strategies Student-interventionist pairings were made such that almost all students designated to receive services through the distance support model had been previously supported by their assigned interventionist in their educational placement. This ensured that rapport and instructional control had already been established. Given that the distance model involved conditions that varied greatly from the previous conditions, interventionists were provided with supplementary training in rapport building, prompting, and reinforcement under the conditions associated with the distance model. Interventionists received training and supervision via the BCBA during distance support sessions at a minimum of once weekly. Overlap support was provided by the BCBA, who joined the virtual session. Immediate feedback to the interventionist was provided using the chat function during the session, and delayed feedback was provided via a performance checklist specific to distance support sessions. Additionally, interventionists participated in weekly training via a virtual meeting platform. It was determined that ongoing training and support at a high rate were essential during the initial weeks of the model to allow for rapid problem resolution and adjustments in procedures given the novelty of the model and the continuously evolving conditions under which it was introduced.

Distance support intervention sessions A general session structure for distance support sessions was developed and further individualized as needed. Sessions were designed to be up to $3 \mathrm{hr}$ in duration, 5 days weekly. Session durations and frequencies were then individualized to meet students' service needs. Ongoing analysis of student data was conducted to determine if adjustments in service levels should be made. The general session structure involved the following: (a) a brief period of rapport building; (b) a 30-s positive statement timing (Calkin and Pennypacker, 2003) during which the student was asked to state positive things about him- or herself, the work he or she did, and/or things that made the student feel happy, joyful, or more confident, followed by the student conducting a positive statement timing for the BI; (c) structured teaching targeting one to two positive replacement skills; (d) a check-in concerning academic work in progress or academic support needs; (e) support toward the completion of required academic work; and (f) a more natural condition under which to target behavior reduction and replacement skill goals (see Appendix D).

The session structure was further differentiated at the level of the student. For some students, and particularly those at the secondary-education level, academic work was completed during a significant portion of the session with the BI's 
support. For other students, a greater portion of the session consisted of structured teaching on replacement skills. Interventionists were given access to the student's Google Classrooms and other instructional platforms utilized by the student's teacher to assist with task completion and submission. At times, interventionists provided support to students during virtual sessions with other service providers, including speech therapists, occupational therapists, and/or resource specialists. During these sessions, the interventionist facilitated student participation via the implementation of behavior and reinforcement protocols. For example, interventionists might prompt the student to attend, respond to questions, or emit target replacement behaviors and might provide reinforcement via a token economy or with subtle social reinforcers including a smile, thumbs-up, or comments through the chat feature. For some students, peer and/or small-group sessions were introduced to target social or peer-related goals, as well as to allow for opportunities for skill development and generalization under conditions more closely matched to those on school campuses (Appendix E).

BCBA supervision and parental support In addition to providing training and conducting overlaps with the interventionists to ensure treatment integrity with respect to individual student protocols and programming, the BCBA overseeing each student's distance support intervention was responsible for acquisition programming related to IEP goals managed by the BCBA, as well as data-based modifications related to the student's behavior intervention and reinforcement protocols. The BCBA also guided and oversaw communication between the parent and interventionist, as well as the interventionist and the student's teacher, case manager, and/or special service providers, related to academic assignments and tasks provided to the student. This is not dissimilar to the role the BCBA maintained prior to school closure; however, the variance in the two models of service delivery did require that the BCBA modify protocols to accommodate for delivery under the conditions of the distance model. The BCBA must also assess the antecedent and consequential events associated with challenging behaviors reported by the parent and/or observed during distance support sessions and make changes to the behavior intervention plan as needed given potentially different maintaining contingencies (i.e., functions) across school and home settings.

Results from parent interviews and surveys were utilized to develop brief, video-based modules related to reported concerns and specific support requests. Video content was developed and delivered by BCBAs, and included developing a student workstation, creating a routine and schedule for instructional activities, prompting hierarchies, building and sustaining rapport, positive reinforcement for desirable behaviors, shaping requests for breaks and assistance, and antecedent strategies to address undesirable behaviors. These videos were very brief (i.e., 5-10 $\mathrm{min}$ ) and allowed parents to access information and strategies related to areas they had requested with the ability to do so at a time workable for them. In addition to providing parents with access to video-based modules, the student's supervising BCBA conducted weekly parent check-ins to address student-specific needs and challenges. Parent check-in meetings focused heavily on antecedent manipulations designed to reduce undesirable behavior during distance support sessions.

\section{Results and Discussion}

The model presented here was developed under critical circumstances, with access to limited educational and support possibilities and under a very abbreviated timeline. Its development and initiation with little delay were rendered crucial in the effort to reduce lapses in student and parental supports during the pandemic in an attempt to limit regression in students' skills. It has currently been implemented for a short period but has yielded some promising outcomes based on reports provided by students and parents, as well as preliminary data on student measures. Additional data are needed to evaluate the effectiveness of distance support models, such as the one outlined here, and to better inform our approach and practices to supporting students in this format. This challenge involves the utilization of measurement and data analysis to inform intervention at the level of the individual student and beyond, and it is a challenge that BCBAs are particularly skilled to approach. Both BCBAs employed by school districts and those serving students through an NPA have the skills to use data to refine how we support students when inperson support is not possible. We encourage each BCBA serving in these roles to fully commit to the challenge of measurement and analysis of the effects of distance-based behavioral intervention and to share findings with both the behavior-analytic and educational communities. Although summer break is rapidly approaching, school districts across the country have already confirmed that extended-school-year services for special education students will be delivered in a distance format. Additionally, it is highly likely that distance education will continue for students at some level into the next school year, with many current proposals involving hybrids of distance and campus-based models and much discussion related to the reliance on distance education if additional outbreaks result in the need for repeated school closures over the course of the next 12-18 months. The data collected now can help better inform practices used during these upcoming periods. We have provided some suggested measures for data that may be collected and analyzed at the level of the individual student and then subjected to analyses across students, programs, distance educational practices, and beyond to move in the direction of a more comprehensive and informed model 
for the future implementation of services (see Appendix F). The process our NPA moved through in the last 2 months, as we desperately attempted to ensure that our students received much-needed services, was informative and helped shape our model and its continued evolution. Information related to what was learned, early results, and recommendations for others faced with similar tasks are addressed in what follows.

\section{The Importance of Collaboration}

Much of the work that was required to implement the model described did not involve simply outlining specific procedural components, but rather the front-end collaboration and advocacy between our organization as the NPA and county- and district-level administrators to secure the continuation of services for students. Collaborative relationships can be tested during uncharted conditions, particularly when each party is responding to multiple contingencies and navigating planning and decision making under novel, high-stakes circumstances. School districts, as well county and state offices of education, are attempting to navigate distance learning for all students, including special education students, while also planning for their own teams to learn how to function in a new paradigm to ensure that children continue to be educated. Their focus, not unlike that of many behavior-analytic organizations (including those that provide NPA services), can be overly internal, and engagement with external agencies, including those that support their students, may not be prioritized during these trying times. It may be difficult to feel heard, given that each party is responding to different and rapidly changing contingencies. In our experience, this led to initial discussions with school districts related to student support during COVID-19 closures to remain unresolved. Early engagements failed to move at the pace deemed necessary to protect students' interests. These less successful collaborative discussions were more open ended in nature. There were many sticking points that were focused highly on the reduction in student supports due to conditions associated with the COVID-19 guidelines, the workability of a distance model, and the interrelated pieces that had not yet been determined at the school-district level. The steps taken by the NPA that supported an agreement involved developing a model with the potential for individualization at the level of each student, proposing the model in a written and consumable format, and scheduling follow-up meetings with school district administrators to discuss the model and its application at the level of individual students. The model was also shared with county-level administrators who recognized the need for NPAs to remain supported by districts during periods of closure given that, with the reopening of schools, NPA services would be sorely needed.

During follow-up meetings with school district administrators, a clear explanation of the content and format of distance behavior intervention sessions was fundamental in securing an agreement. It is recommended that other agencies advocating for the continuation of services prepare and share their descriptions of a distance or telepractice model in a variety of formats, such as bulleted written descriptions, simple oral descriptions, and video models of a mock or actual session. It is further recommended that we rely on data obtained via collaborative sharing outlets to help secure services for students. It was our experience that levels of service delivery — and particularly the level we were proposing - were often refuted based on assumptions that a family and/or student might not be able to "handle" a structured, daily distance support session. Although it is critical that service levels be differentiated as needed to meet the student's and parents' current situation and needs, it is important that decreases in services outlined in the student's IEP are not offered based on assumptions, in the absence of directly engaging families and soliciting their feedback and preferences. Our students have received approximately $15 \mathrm{hr}$ weekly of distance support over the course of an 8-week period. Of the 24 students receiving these services, attendance averaged $98 \%$ for all weeks (range 84\%-100\%). These data, coupled with parent survey data, suggest commitment and satisfaction in the presence of a relatively intensive distance support model.

\section{Social Validity}

In the development and implementation phases of the presented model, parent feedback related to their experiences during the closure was gathered via interview and surveys. These data were summarized and shared using a simple format with county- and district-level school administrators. Parents reported that their children needed continuous support during the instruction they attempted at home, that they were dealing with high rates of undesirable behavior, and that their children lacked many of the skills needed to participate in virtual conferences with teachers and/or with the instructional paper packets that had been provided. Parents requested "tips" for treating challenging behavior and for how to help their child with schoolwork and "increase motivation." Parents asked for coaching and whether engagement between the student and his or her BI was possible during the closure. This information not only contributed to the specific components of the model (e.g., parental support) but also helped facilitate collaboration with school districts. Subjective parental reports (i.e., social validity) can be critical as a starting point under novel circumstances and when determining how to best support students, families, and one another. In addition to the initial surveys completed with parents, ongoing surveys have been administered to parents in relation to their child's response to services and their specific needs and supports for themselves and their child. Whenever possible, it is also important to engage students in identifying and sharing what supports are working for them. We recommend this be done in a variety of ways but that some involve repeated, standard measures to allow for 
comparison and evaluation. Overall, students shared that they appreciated the levels of BI support, were interested in focusing sessions on completing academic work, and wanted assistance with managing their behavior and finding ways to connect with others. More specifically, $70 \%$ of students reported that the services were very helpful, and nearly $90 \%$ reported that they enjoyed most to all sessions. Parent survey data indicated that $92 \%$ of respondents found services to be very helpful. Data such as these can be employed to make adjustments and additions to support services at the level of the model and the individual student.

\section{Student Readiness}

When considering the generalizability of our model, it is worth noting that the majority of our students were in general education placements with full-time BI support. These students demonstrated attending, instruction-following, and tracking skills that allowed them to participate in distance support intervention sessions. For students who demonstrated these skills less consistently, acquisition programs were introduced to shape these prerequisite skills. There are many special education students whose skill deficits and/or rate, topography, and/or severity of undesirable behavior might impact the effectiveness of the model detailed here. These are the students for whom active collaboration between school districts, BCBAs, and/or other supporting agencies may be even more essential. For these students, we should still move forward with creating preparedness for distance education. Again, we are uncertain how long distance education will be needed and/or if it will be necessary again in the future. For these reasons, steps should be taken to shape prerequisite skills for all students. Assessment of student deficits that are considered barriers to their participation in distance education can lead to programming and intervention designed to address these deficits. For example, if the student demonstrates limited attending to the screen, programming designed to shape these skills should be introduced. If a student will sit and/or participate for only very brief periods during distance support sessions, protocols related to reinforcing gradually increasing durations of sitting and engagement in these sessions should be introduced. A student's skill deficits should not be used as a rationale to reduce or eliminate a student's access to telepractice models (see Appendix G). Instead, it is critical to identify these deficits, establish baseline performances, and target the development of skills that will promote the student's access to instruction. There is no single format that will be a viable option for all special education students, but all students are entitled to and should be provided with access to their educational programs to the fullest extent possible. It is imperative that we think outside the box, probe, collect data, and make revisions and decisions quickly to ensure that all special education students are well supported during this and future crises. Behavior analysts are particularly well suited for this level of problem resolution given our repertoires in the areas of identifying component and composite skills, breaking down tasks into small units, measurement, and data analysis. This is a time when barriers should not be identified as a rationale to provide fewer supports or less service, but rather for agencies to work incessantly as a collaborative team to address each student's needs.

Another consideration is that not all students have access to the technology and equipment used for distance models that involve Internet platforms. This was not an issue for students served in this model, as their school district made available Chromebooks and air cards for Internet connectivity to students in need. The degree to which this practice is standard across school districts is unknown, and yet it is critical that school districts address issues related to access and equity for all students.

\section{Conclusion}

There is no manual or specific set of practice guidelines that details exactly how we can support our students, parents, and teams during this crisis. We can, however, make a commitment to each and every student that the support services deemed necessary for them to access their educational programs will be implemented to the greatest extent and with as little delay as possible during this time. We can commit to rejecting proposals that a student's skill repertoire, behavioral excesses, and/or physiological condition should result in a service plan that involves reduced or no services. We can commit to continuing to explore possibilities to develop new and varied models for service delivery, for resolving problems, and for measuring student performance and obtaining repeated parent and student input concerning their needs and the workability of what is implemented. We can commit to data analysis and to modifications in service level, mode, and delivery with little haste when the data suggest that the student is not making progress toward goals. This is the time for us to wrap our support around our students, their families, and our collaborative teams as a means to pave a successful path back to learning together on school campuses in the near future.

Funding Not applicable.

\section{Compliance with Ethical Standards}

Conflict of Interest There were no conflicts of interest for any of the authors involved in this research. 


\section{References}

California Department of Education. (2020). New COVID-19 guidance for K-12 schools. Retrieved April 8, 2020, from http://www.cde.ca. gov/ls/he/hn/guidance.asp

Calkin, A. B., \& Pennypacker, H. S. (2003). A minute a day makes good feelings grow. European Journal of Behavior Analysis, 4(1-2), 512.

Ferguson, J., Craig, E. A., \& Dounavi, K. (2019). Telehealth as a model for providing behaviour analytic interventions to individuals with autism spectrum disorder: A systematic review. Journal of Autism and Developmental Disorders, 49(2), 582-616.

Hanley, G. P., Jin, C. S., Vanselow, N. R., \& Hanratty, L. A. (2014). Producing meaningful improvements in problem behavior of children with autism via synthesized analyses and treatment. Journal of Applied Behavior Analysis, 47(1), 16-36.

Individuals With Disabilities Education Act, 20 U.S.C. § 1400 (2004)

National Center for Education Statistics (2020). The condition of education. Retrieved April 8, 2020, from https://nces.ed.gov/programs/ coe/indicator_cgg.asp.
Peele, H., \& Riser-Kositsky, M. (2020). Coronavirus and school closures. Education Week. Retrieved April 8, 2020, from https://www. edweek.org/ew/section/multimedia/map-coronavirus-and-schoolclosures.html

Rodriguez, K. A. (2020). Maintaining treatment integrity in the face of crisis: A treatment selection model for transitioning direct ABA services to telehealth. Behavior Analysis in Practice.

Tomlinson, S. R., Gore, N., \& McGill, P. (2018). Training individuals to implement applied behavior analytic procedures via telehealth: A systematic review of the literature. Journal of Behavioral Education, 27(2), 172-222.

U.S. Department of Education. (2020). COVID-19 (“coronavirus") information and resources for schools and school personnel. Retrieved April 8, 2020, from https://www.ed.gov/coronavirus

Publisher's Note Springer Nature remains neutral with regard to jurisdictional claims in published maps and institutional affiliations. 